\title{
POLUIÇÃO QUÍMICA RELACIONADA AO AR DE INTERIORES NO BRASIL
}

\section{Adriana Gioda}

Departamento de Química, Universidade da Região de Joinville, Joinville - SC

Francisco Radler de Aquino Neto*

Instituto de Química, Universidade Federal do Rio de Janeiro, CT, Bloco A, Ilha do Fundão, 21949-900 Rio de Janeiro - RJ

Recebido em 23/4/02; aceito em 4/9/02

\begin{abstract}
CHEMICAL POLLUTION RELATED TO THE INDOOR AIR IN BRAZIL. During the last two decades there has been increasing concern within the scientific community related to the effects of indoor air quality on health. Changes in building design devised to improve energy efficiency and new synthetic building materials have contributed to increase pollutant concentrations. These factors have generated poor air quality and caused the development of a lot of symptoms known as Sick Building Syndrome (SBS). In Brazil, there are few studies in this area, collected in this review to support researches and awareness of the need to adopt preventive measures to reach a better quality for the Indoor Environment.
\end{abstract}

Keywords: indoor air quality; VOCs; indoor environment.

\section{INTRODUÇÃO}

A Qualidade do Ar de Interiores (QAI) é uma ciência nova surgida na década de 70, quando houve escassez de energia nos países desenvolvidos. Como maneira de racionalizar a energia, surgiram os edifícios selados. Esses ambientes, devido à baixa troca de ar interno/externo, somada a diversos produtos como forração, acabamento e mobiliário que contém vários tipos de substâncias químicas, passaram a ter elevados níveis de poluentes. Esse fato gerou um aumento significativo no número de queixas relacionados com a qualidade do ar em ambientes fechados, principalmente em países localizados em clima frio. Os sintomas relacionados à qualidade do ar passaram a ser mais estudados e, atualmente, são conhecidos como Síndrome dos Edifícios Doentes (SED), a qual é reconhecida pela Organização Mundial da Saúde (OMS) desde o início da década de $80^{1}$.

De acordo com a $\mathrm{OMS}^{1}$, os sintomas mais comuns de SED são irritação e obstrução nasal, desidratação e irritação da pele, irritação e secura na garganta e nas membranas dos olhos, dor de cabeça, letargia e cansaço generalizado levando à perda de concentração. Podem ser percebidos um ou mais sintomas, que geralmente desaparecem quando a pessoa permanece por um tempo longo fora desse ambiente.

São inúmeras as fontes de poluentes em ambientes internos, além das atividades ocupacionais do próprio homem. Dentre os contami- nantes usualmente monitorados estão o dióxido de carbono $\left(\mathrm{CO}_{2}\right)$, o monóxido de carbono (CO), os compostos orgânicos voláteis totais e especiados (COVsT e COV), os compostos orgânicos semi-voláteis (COSVs), matéria particulada, nicotina e microorganismos ${ }^{2}$. A Tabela 1 mostra as fontes mais comuns desses compostos.

No Brasil são poucos os registros relacionando QAI e SED ${ }^{4}$. O fato do Brasil ter um clima subtropical não torna necessário o uso constante de sistemas de refrigeração e, menos ainda, de calefação. Devido a isso, muito pouco tem sido estudado nessa área. Porém, há uma tendência em construir prédios selados devido a vários fatores como estética, climatização e menor ruído, com isso, faz-se necessário um sistema de ar condicionado central. É possível que essa nova tendência acarretará em um aumento do número de casos de SED no país.

O governo está ficando alerta ao fato e, visando minimizar os efeitos à saúde, o Ministério da $S$ aúde ${ }^{5}$ publicou a Portaria 3523 de 28 de agosto de 1998, contendo Regulamento Técnico que visa "promover o estabelecimento de medidas referentes à limpeza dos sistemas de climatização e medidas específicas de padrões da qualidade do ar identificando poluentes de natureza física, química e biológica com suas respectivas fontes, visando a prevenção de riscos à saúde dos ocupantes desses ambientes". A Agência Nacional de Vigilância Sanitária, em decorrência da Portaria 3523, publicou a Resolução

Tabela 1. Fontes típicas de poluição do ar em ambientes internos ${ }^{2,3}$

\begin{tabular}{ll}
\hline Poluente & Maior fonte de emissão \\
\hline Dióxido de carbono & atividade metabólica, atividades de combustão, veículos motores em garagem \\
Monóxido de carbono & queima de combustível fóssil, aquecedores a gás ou querosene, fogão, fumaça de cigarro \\
Formaldeído & materiais de construção e mobiliário \\
COVs & $\begin{array}{l}\text { adesivos, solventes, materiais de construção, volatilização, combustão, pintura, fumaça de cigarro; atividades } \\
\text { de limpeza; fotocopiadoras, impressoras a laser } \\
\text { Partículas }\end{array}$ \\
\hline
\end{tabular}

*e-mail: radler@iq.ufrj.br 
176 de 24 de outubro de $2000^{6}$ (ANVISA, 2000; atualmente em revisão) com algumas orientações técnicas sobre "Padrões Referenciais da Qualidade do Ar de Interiores em ambientes climatizados artificialmente de uso público e coletivo". Essa Resolução é revolucionária, pois define os parâmetros mínimos para uma boa qualidade do ar de interiores como a concentração de $\mathrm{CO}_{2}$ e material particulado, temperatura, umidade relativa e velocidade do ar. Parâmetros mais complexos como COVs e aldeídos necessitam ser melhor estudados para que sua influência sobre os ocupantes possa ser quantificada e padrões estabelecidos.

Aquino Neto e Brickus ${ }^{7}$ sugeriram valores máximos para contaminantes presentes em ar de ambientes internos. Esses valores foram baseados em recomendações de organismos internacionais e nacionais adaptados à realidade brasileira. Nesse aspecto, características climáticas, sócio-econômicas, estruturais e geográficas do Brasil foram consideradas, bem como a matriz energética do país, nossa arquitetura, decoração e costumes.

Regulamentações de saúde ocupacional relativa às atividades industriais continuam a ser uma atividade do Ministério do Traba1 ho ${ }^{8}$, através do Decreto-Lei 5452 de 1943 estabelecidas na NR-15. Esse tem baseado suas regulamentações nos valores de limite de tolerância publicados pela ACGIH (USA). Os limites de exposição em $\mathrm{mg} / \mathrm{m}^{3}$ são baseados em $48 \mathrm{~h} / \mathrm{semana}$ no Brasil e $40 \mathrm{~h} / \mathrm{semana}$ nos EUA. O modo como a NR-15 foi estabelecida gera alguns questionamentos sobre sua abrangência. Primeiro, com relação à equivalência de muitos padrões. O Brasil permite altas exposições a certas substâncias através de padrões permissíveis ou completa ausência de padrões. Esses padrões embora sigam a ACGIH, não são atualizados como a mesma. Com exceção do benzeno, os demais permanecem com os mesmos valores desde a criação da norma. Além disso, vários compostos carcinogênicos não são regulamentados no Brasil. Como a maioria dos compostos não foram submetidos a todos os testes de toxidez necessários é possível que, tanto no setor industrial quanto em ambientes interiores, estejamos expostos a uma carga mais elevada de poluentes do que seria recomendável ${ }^{4}$.

Os parâmetros não definidos pela Resolução $176^{6}$, seguem outros padrões nacionais como o CONAMA $^{9}$ ou padrões internacionais como $\mathrm{NIOSH}^{10}{ }^{\mathrm{USEPA}^{11}}{ }^{1}$ OSHA $^{12} \mathrm{e} \mathrm{WHO}^{13}$, esses apenas com fins comparativos.

\section{POLUIÇÃO QUÍMICA NO AR DE INTERIORES NO BRASIL}

Os trabalhos pioneiros no Brasil foram iniciados em 1992, através de uma colaboração entre o Laboratório de Apoio ao Desenvolvimento Tecnológico (LADETEC) da Universidade Federal do Rio de Janeiro e o Laboratório de Aerossóis e Gases Atmosféricos (LAGA) do Instituto de Química da Universidade de São Paulo (o qual encerrou suas atividades em 1995). Em 1997, o laboratório de toxicologia localizado no CESTEH-ENSP-FIOCRUZ/RJ criou um programa da qualidade do ar de interiores que continua operando até o momento.

No ano de 1995, embora com poucos profissionais trabalhando nessa área, foi criada a Sociedade Brasileira de Meio Ambiente e Controle da Qualidade do Ar de Interiores - BRASINDOOR (www.brasindoor.com.br). Esta sociedade tem sido responsável pela divulgação (através de congressos, cursos, publicações, etc.) da maioria dos trabalhos realizados no Brasil referentes à QAI.

O presente trabalho pretende fazer um levantamento para avaliar a situação atual do Brasil frente a esse problema.

Uma das primeiras pesquisas realizadas no Brasil foi em $1990^{14}$ onde os níveis de carbonila foram registrados em 3 locais em Salvador, Bahia. Nesse estudo foi enfocada a relação entre os níveis de acetaldeído e o uso de etanol como combustível. Também foram es- tudados os níveis de aldeídos e carbono total no Museu de Arte Contemporânea de São Paulo. Nesses estudos a relação I/E sugeria importantes fontes internas desses poluentes ${ }^{15,16}$.

Outros trabalhos apresentados por Miguel et al. ${ }^{17-19}$ e Allen et $a l .{ }^{20}$ avaliaram ambientes de escritórios, hotéis e restaurantes nas cidades de São Paulo, Rio de Janeiro e Campinas. A pesquisa detectou níveis de vários poluentes acima ou próximo aos limites fixados pelas recomendações internacionais. Foram medidos os níveis de poluentes em material particulado e na fase gasosa, simultaneamente. Partículas suspensas respiráveis (PST) e carbono total respirável (CTR) apresentaram maiores concentrações internas (I) que externas (E). A relação das concentrações I/E sugere que cloreto, nitrato e potássio associados a aerossol são fontes poluidoras de ambientes internos. As medidas de CTR sugerem também fontes internas de poluição, bem como, a formação in situ de ácido nitroso. As concentrações de ácido acético e ácido fórmico foram muito mais elevadas em vários ambientes internos. Deposição a seco foi observada em ambientes internos para ácido clorídrico, ácido nítrico e dióxido de enxofre. Nesses mesmos locais Santos e colaboradores ${ }^{21}$ avaliaram COVs. Em todos os locais estudados o nível de muitos poluentes foi maior no ar interno que no ar externo, sugerindo fontes internas de poluição.

Em dezembro de 1997, Silva e colaboradores ${ }^{22}$ realizaram amostragens de compostos orgânicos voláteis e semivoláteis durante a preparação de almoço utilizando lenha como combustível. A cozinha amostrada estava localizada no município de Carandaí, MG, em zona rural. As amostragens foram realizadas em três etapas: antes, durante e após cozinhar. Também foram realizadas amostragens externas. No ar externo foram detectados primariamente terpenos e a concentração de COVsT foi de $13,8 \mu \mathrm{g} / \mathrm{m}^{3}$. Na amostragem antes de iniciar qualquer atividade foram observados níveis baixos de COVs. Na segunda etapa, durante a preparação do almoço, foi observada maior concentração de COVsT. Na terceira etapa observou-se um decréscimo nos níveis de COVs. Com esses dados foi possível observar que o ato de cozinhar eleva os níveis de COVs. Outro trabalho avaliou o nível de exposição a $\mathrm{NO}_{2}$ em cozinhas que utilizam GLP. Ugucione e colaboradores ${ }^{23}$ encontraram uma concentração 30 vezes maior desse poluente quando o GLP está sendo queimado.

Oliveira e colaboradores ${ }^{24,25}$ realizaram amostragens do ar no interior e exterior do Museu Nacional de Belas Artes, no centro do Rio de Janeiro. Os resultados analíticos da concentração de acetaldeído no ar variaram de 11,6 a $39,7 \mu \mathrm{g} / \mathrm{m}^{3}$ enquanto que as concentrações dos COVsT variaram de 91 a $3970 \mu \mathrm{g} / \mathrm{m}^{3}$. Os dados mostraram que as fontes de todos os compostos eram externas ao edifício.

Leite e colaboradore ${ }^{26}$ avaliaram BTX em vários locais no Rio de Janeiro. A concentração média de BTX encontrada para escritório foi de $24,4 \mu \mathrm{g} / \mathrm{m}^{3}$ para benzeno, $200,3 \mu \mathrm{g} / \mathrm{m}^{3}$ para tolueno e $40,4 \mu \mathrm{g} / \mathrm{m}^{3}$ para xilenos. Para o ar urbano a concentração foi de $8,5 \mu \mathrm{g} / \mathrm{m}^{3}$ para benzeno, $37,6 \mu \mathrm{g} / \mathrm{m}^{3}$ para tolueno e $11,9 \mu \mathrm{g} / \mathrm{m}^{3}$ para xilenos. Em locais industriais os valores encontrados foram de $3,9 \mathrm{mg} / \mathrm{m}^{3}$ para benzeno, $51,1 \mathrm{mg} / \mathrm{m}^{3}$ para tolueno e $496,3 \mathrm{mg} / \mathrm{m}^{3}$ para xilenos. Os valores de BTX medidos no escritório e ar urbano mostram que as pessoas podem estar expostas a concentrações de BTX prejudiciais à saúde.

Brickus e Moreira ${ }^{27}$ avaliaram a qualidade do ar em uma pista semi-aberta de kart. A pista amostrada está localizada na cidade do Rio de Janeiro em uma área arborizada, distante do tráfego. As amostragens foram realizadas em duas etapas, antes e durante as atividades. Os resultados mostraram antes de iniciar as atividades, níveis de COVsT de $11,4 \mu \mathrm{g} / \mathrm{m}^{3}$ no ar interno, $19,6 \mu \mathrm{g} / \mathrm{m}^{3}$ no ar externo e BTX de $6,1 \mu \mathrm{g} / \mathrm{m}^{3}$ no ar interno e $7,4 \mu \mathrm{g} / \mathrm{m}^{3}$ no ar externo. As amostragens durante as atividades indicaram valores de $111,1 \mu \mathrm{g} / \mathrm{m}^{3}$ 
para COVsT e $22,1 \mu \mathrm{g} / \mathrm{m}^{3}$ para BTX no ar interno, um aumento considerável de COVsT durante a corrida.

Em agosto de 1996, Brickus e colaboradore ${ }^{28}$ fizeram amostragens de COVsT, nicotina, MPI, e aldeídos no ar interno e externo em um edifício no centro do Rio de Janeiro, cujos ocupantes fizeram reclamações da QAI. Os resultados mostraram que os teores dos poluentes amostrados foram mais elevados no ambiente interno que no externo. Os COVsT apresentaram índices preocupantes, $1000-5000 \mu \mathrm{g} / \mathrm{m}^{3}$, bem acima da recomendação internacional $\left(300 \mu \mathrm{g} / \mathrm{m}^{3}\right)^{29}$. Após adotar medidas remediativas nova amostragem foi realizada ${ }^{30} \mathrm{em}$ agosto de 1998. Os resultados obtidos nessa segunda amostragem mostram uma melhora significativa na qualidade do ar do prédio referente, principalmente, aos COVsT.

Brickus e colaboradores ${ }^{31}$ avaliaram a QAI em um edifício de escritórios no Rio de Janeiro. Os pontos de amostragem compreenderam três escritórios localizados na mesma coluna vertical do prédio ( $1^{\circ}, 13^{\circ}$ e $25^{\circ}$ andares). Foram analisados COVsT, MPT, aldeídos, nicotina e partículas inaláveis em suspensão. As concentrações de acetaldeído foram maiores que as de formaldeído. As concentrações de COVsT estavam na faixa de 304,3 a $1679,9 \mu \mathrm{g} / \mathrm{m}^{3}$ para o ar interno e 22 a $643,2 \mu \mathrm{g} / \mathrm{m}^{3}$ para o externo. A relação I/E para COVsT foi maior que 1 , o que indica que os COVs encontrados no ar interno são originados principalmente de fontes internas ou há mecanismos de concentração. Nesse mesmo prédio foi avaliado de forma diferenciada um escritório ( $9^{\circ}$ andar) que estava sendo reformado ${ }^{32-34}$. Durante a renovação (dezembro de 1995) as concentrações de COVsT estavam na faixa de $688,8-1.089,9 \mu \mathrm{g} / \mathrm{m}^{3}$ para o ar interno e $215,5 \mu \mathrm{g} / \mathrm{m}^{3}$ para o ar externo, enquanto que formaldeído apresentou uma concentração de $92,8 \mu \mathrm{g} / \mathrm{m}^{3}$ e acetaldeído de $4,14 \mu \mathrm{g} / \mathrm{m}^{3}$. Durante a ocupação do escritório (fevereiro de 1996) os níveis internos ficaram entre $545,4-625,6 \mu \mathrm{g} / \mathrm{m}^{3}$ e $132,8-188,0 \mu \mathrm{g} / \mathrm{m}^{3}$ para os externos. Porém, as concentrações de formaldeído $\left(114,1 \mu \mathrm{g} / \mathrm{m}^{3}\right)$ e acetaldeído $\left(11,5 \mu \mathrm{g} / \mathrm{m}^{3}\right)$ aumentaram. Concentrações de COVsT estiveram na faixa de 446,2-638,0 $\mu \mathrm{g} / \mathrm{m}^{3}$, formaldeído de $63,4 \mu \mathrm{g} / \mathrm{m}^{3}$, acetaldeído de $37,6 \mu \mathrm{g} / \mathrm{m}^{3}$, um mês após a ocupação. A última avaliação realizada, após 6 meses, mostrou uma concentração em torno de $350 \mu \mathrm{g} / \mathrm{m}^{3}$ para COVsT, $74,5 \mu \mathrm{g} / \mathrm{m}^{3}$ para formaldeído e $23,9 \mu \mathrm{g} / \mathrm{m}^{3}$ para acetaldeído. Concentrações internas de BTX foram medidas na primeira avaliação e 6 meses mais tarde. Os resultados mostram também uma redução desses compostos.

Pires e Carvalho ${ }^{35}$ estudaram a presença de compostos carbonílicos de baixo peso molecular (C1-C4) e glutaraldeído em 14 locais amostrados (laboratório, museu, hospital, escritório, residência, escola) em São Paulo, em julho de 1997. Formaldeído foi a espécie mais abundante (29 ppb), seguido por acetona e acetaldeído ( 25 e 17 ppb), com uma pequena contribuição de propanal, crotonaldeído e isômeros C4 ( 0,7 a 1,5 ppb).

Pereira e colaboradore ${ }^{36}$ propuseram uma nova metodologia para análise de aldeídos em ambientes internos e externos. Para testar essa metodologia foram utilizados alguns locais dentro da própria instituição. Os valores encontrados variaram entre $23,0-84,0 \mu \mathrm{g} / \mathrm{m}^{3}$. Foram observados valores relativamente altos para os laboratórios.

Alguns trabalhos foram desenvolvidos na própria FIOCRUZ quando implantados os estudos de QAI. Nessa oportunidade foram avaliados vários ambientes na própria instituição como creche, biblioteca e salas ${ }^{37-39}$.

No I Congresso Internacional de Qualidade do Ar de Interiores realizados pela BRASINDOOR no Rio de Janeiro, em março de 2001, foram apresentados os mais recentes trabalhos na área de QAI no Brasil, que serão publicados na Revista BRASINDOOR.

$\mathrm{O}$ trabalho realizado por Carneiro e Aquino $\mathrm{Neto}^{40}$ avaliou a qualidade do ar em uma caixa-forte de um banco comercial na cidade do Rio de Janeiro. Os parâmetros estudados foram os COVs, nicotina, aldeídos, MPT, além de fungos e bactérias. Não foi observado nenhum local interno crítico com relação aos contaminantes individualmente, na coleta de 2000. Um fator interessante foi a presença de diclorobenzeno, provavelmente proveniente de inseticidas. Nova amostragem foi realizada em $2001^{41}$, sendo observado um aumento de COVsT $\left(1272 \mu \mathrm{g} / \mathrm{m}^{3}\right)$ comparado à coleta de $2000\left(321-398 \mu \mathrm{g} / \mathrm{m}^{3}\right)$.

Gioda e Aquino $\mathrm{Neto}^{42}$ realizaram uma avaliação química e microbiológica em uma gráfica no Rio de Janeiro, em abril de 2000. Após a investigação foi diagnosticado um elevado teor de (COVsT). Em março de 2001, após implementação de medidas remediativas foi realizada nova amostragem de contaminantes químicos na gráfica. Os índices de COVsT foram reduzidos de 40 a $80 \%$ porém, ainda, continuavam elevados, ultrapassando os limites nacionais $\left(500 \mu \mathrm{g} / \mathrm{m}^{3}\right)^{7} \mathrm{e}$ internacionais $\left(300 \mu \mathrm{g} / \mathrm{m}^{3}\right)^{29}$. Os demais parâmetros apresentaram algumas alterações, mas mantiveram-se dentro dos limites toleráveis ${ }^{43}$.

Almeida e colaboradores ${ }^{44,45}$ avaliaram a qualidade do ar de interiores em sete cidades no Estado do Rio de Janeiro. Os resultados obtidos mostram que as concentrações de COVsT foram da ordem de $800 \mu \mathrm{g} / \mathrm{m}^{3}$, benzeno de $10 \mu \mathrm{g} / \mathrm{m}^{3}$, tolueno de $200 \mu \mathrm{g} / \mathrm{m}^{3}$, xileno de $30 \mu \mathrm{g} / \mathrm{m}^{3}$, formaldeído de $40 \mu \mathrm{g} / \mathrm{m}^{3}$ e MPT de $48 \mu \mathrm{g} / \mathrm{m}^{3}$ nos pontos mais críticos. Esses resultados demonstram um preocupante índice de poluentes em residências.

Costa e Moreira ${ }^{46}$ avaliaram a exposição ao benzeno decorrente do hábito de fumar, em trabalhadores de escritórios administrativos, climatizados artificialmente na cidade do Rio de Janeiro. Os resultados mostraram que a exposição individual ao benzeno entre os trabalhadores fumantes (25,3 ppb) e os não fumantes (13,0 ppb) apresentou diferença significativa. A contribuição de benzeno no ar interno foi menor $(8,4 \mathrm{ppb})$ que aquela observada na área de respiração dos trabalhadores.

Silveira e colaboradores ${ }^{47}$ avaliaram alguns parâmetros da qualidade do ar no interior de um Terminal Aeroportuário na Cidade do Rio de Janeiro, no verão e no inverno, em diferentes períodos. Os resultados encontrados variaram entre $16,2-283,1 \mu \mathrm{g} / \mathrm{m}^{3}$ para MPT e 51-1878 $\mu \mathrm{g} / \mathrm{m}^{3}$ para COVsT.

Bortolli e Pires ${ }^{48}$ desenvolveram uma nova metodologia para análise de formaldeído e aplicaram a ambientes internos não industriais em Porto Alegre. Os resultados obtidos indicaram concentrações variando entre 10 e 740 ppb. A concentração média no ar externo (10 ppb) foi inferior ao ar externo, indicando fontes internas de poluição.

Mattos e colaboradores ${ }^{49}$ avaliaram a exposição ao chumbo em trabalhadores de 4 fábricas reformadoras de baterias no Rio de Janeiro. Os resultados do monitoramento ambiental realizado nas fábricas e reformadoras indicam altos níveis de exposição $(0,068$ a $0,802 \mathrm{mg} / \mathrm{m}^{3}$ ) com concentração média de $0,302 \mathrm{mg} / \mathrm{m}^{3}$, acima do limite de exposição ambiental $\left(0,1 \mathrm{mg} / \mathrm{m}^{3}\right)^{49}$.

Parreira e colaboradores ${ }^{50}$ monitoraram acetona e sete aldeídos no ar externo e interno de uma unidade de monitoramento da qualidade do ar de uma sala climatizada do CETEC. Os resultados indicaram pouca influência do ar externo sobre o ar interno.

A Tabela 2 mostra como ainda são poucos os trabalhos publicados ou apresentados em congressos científicos referentes à QAI no Brasil, sendo estes originários basicamente do Rio de Janeiro, seguido por São Paulo.

Os valores encontrados nas pesquisas realizadas mostram dados preocupantes pois, a maioria dos poluentes apresentou uma razão I/ E superior a 1, indicando a presença de fontes internas de poluição. Na Tabela 3, é possível observar com mais clareza esse fato.

Para avaliação de ambientes não industriais no Brasil, para fins legais, segue-se a Resolução $176^{6}$ e/ou CONAMA ${ }^{9}$. Em alguns casos utilizam-se os limites sugeridos pela NR-15. Por não serem oficiais, os limites sugeridos por Aquino Neto e Brickus ${ }^{7}$, embora se- 
Tabela 2. Bibliografia sobre a Qualidade do Ar de Interiores no Brasil

\begin{tabular}{|c|c|c|c|c|c|c|}
\hline \multirow[t]{2}{*}{ Local } & \multirow[t]{2}{*}{ Poluentes } & \multicolumn{2}{|c|}{ Interno } & \multicolumn{2}{|c|}{ Externo } & \multirow[t]{2}{*}{ Referêncic } \\
\hline & & Média $\left(\mu \mathrm{g} / \mathrm{m}^{3}\right)$ & Faixa $\left(\mu \mathrm{g} / \mathrm{m}^{3}\right)$ & Média $\left(\mu \mathrm{g} / \mathrm{m}^{3}\right)$ & Faixa $\left(\mu \mathrm{g} / \mathrm{m}^{3}\right)$ & \\
\hline \multirow{6}{*}{$\begin{array}{l}\text { 1. Escritórios, restaurantes } \\
\text { e hotéis - SP, RJ, } \\
\text { Campinas }\end{array}$} & Nicotina & 0,37 & $0,09-0,69$ & $\mathrm{Nd}$ & - & \multirow{6}{*}{$18,19,21$} \\
\hline & UV-MPI & 5,1 & $1,6-8,5$ & 1,9 & $0,9-3,9$ & \\
\hline & Formaldeído & 20,4 & $6,1-60,7$ & 10,7 & $4,7-27,7$ & \\
\hline & Acetaldeído & 38,3 & $6,1-69,1$ & 33,8 & 9,3-178,0 & \\
\hline & COVsT & & $6,88-568,0$ & & $6,38-138,11$ & \\
\hline & MPI & 95,1 & $33,0-408,0$ & 66,9 & $32,6-145,0$ & \\
\hline \multirow{15}{*}{$\begin{array}{l}\text { 2. Escritórios, restaurantes } \\
\text { e hotéis - SP, RJ, } \\
\text { Campinas }\end{array}$} & MPT & 75,6 & NI & 53,3 & NI & \multirow{15}{*}{17,20} \\
\hline & CRT & 55,1 & NI & 27,7 & NI & \\
\hline & Cálcio & 0,82 & $0,18-3,0$ & 0,95 & $0,18-2,7$ & \\
\hline & Sulfato & 0,30 & $0,18-4,5$ & 0,49 & $0,3-4,9$ & \\
\hline & Nitrato & 0,28 & $0,05-3,7$ & 0,79 & $0,19-2,8$ & \\
\hline & Acetato & 0,19 & $0,06-0,27$ & 0,11 & $0,03-0,29$ & \\
\hline & Potássio & 0,11 & $0,05-1,98$ & 0,08 & $0,06-0,51$ & \\
\hline & Cloreto & 0,10 & $0,03-1,34$ & 0,06 & $0,01-0,13$ & \\
\hline & Sódio & 0,09 & $0,05-0,32$ & 0,11 & $0,03-0,26$ & \\
\hline & Magnésio & 0,06 & $0,02-0,24$ & 0,08 & $0,02-0,24$ & \\
\hline & Formiato & 0,03 & $0-0,013$ & 0,04 & $0-0,12$ & \\
\hline & Amônio & 0,03 & $0,01-1,28$ & 0,06 & $0,02-1,44$ & \\
\hline & Oxalato & 0,02 & $0,01-0,036$ & 0,04 & $0,02-0,42$ & \\
\hline & Piruvato & 0 & 0 & 0 & 0 & \\
\hline & Nitrito & 0 & 0 & 0 & 0 & \\
\hline \multirow[t]{4}{*}{ 3. Cozinha $-\mathrm{MG}$} & COVsT & & & 13,8 & & \multirow{4}{*}{22} \\
\hline & Benzeno & 8,6 & & & & \\
\hline & Hexano & 20,6 & & & & \\
\hline & Nonanal & 7,0 & & & & \\
\hline \multirow{2}{*}{$\begin{array}{l}\text { 4. Museu Nacional } \\
\text { de Belas Artes - RJ }\end{array}$} & Acetaldeído & & $11,6-39,7$ & & & \multirow[b]{2}{*}{24,25} \\
\hline & COVsT & & $91,0-3970,0$ & & & \\
\hline \multirow[t]{3}{*}{ 5. Escritório - RJ } & Benzeno & 24,4 & $6,4-54,8$ & 8,5 & $2,0-10,3$ & \multirow{3}{*}{26} \\
\hline & Tolueno & 200,3 & $62,1-381,4$ & 37,6 & $4,3-129,0$ & \\
\hline & Xileno & 40,4 & $2,9-72,4$ & 11,9 & $1,8-28,1$ & \\
\hline \multirow[t]{3}{*}{ 6. Local industrial } & Benzeno $\left(\mathrm{mg} / \mathrm{m}^{3}\right)$ & 3,9 & $0,07-10,7$ & & & \multirow[t]{3}{*}{26} \\
\hline & Tolueno $\left(\mathrm{mg} / \mathrm{m}^{3}\right)$ & 51,1 & $0,04-201,2$ & & & \\
\hline & Xileno $\left(\mathrm{mg} / \mathrm{m}^{3}\right)$ & 496,3 & $284,3-1.117,0$ & & & \\
\hline \multirow[t]{2}{*}{ 7. Pista de kart - RJ } & COVsT & & $11,4-111,1$ & 19,6 & & \\
\hline & BTX & & $7,4-22,1$ & 6,1 & & 27 \\
\hline 8. Prédio Centro - RJ & Nicotina & 1,7 & $0,3-4,3$ & ND & ND & \\
\hline & Formaldeído & 33,2 & $5,0-48,9$ & 7,1 & $0,3-30,3$ & 28 \\
\hline & Acetaldeído & 44,1 & $5,8-59,3$ & 10,9 & $0,8-49,8$ & \\
\hline & COVsT & 1.052 & $591,0-2.306,0$ & 80,0 & $57,0-113,0$ & \\
\hline & MPI & 32,5 & $14,5-43,7$ & 23,9 & $19,6-30,3$ & \\
\hline 9. Prédio Centro RJ - & Nicotina & 1,05 & $0,6-1,6$ & 0,06 & ND-0,3 & \\
\hline reavaliação & Formaldeído & 41,2 & $18,1-74,4$ & 14,4 & $9,3-18,6$ & \\
\hline & Acetaldeído & 27,0 & $17,7-33,6$ & 17,3 & $8,6-22,7$ & 30 \\
\hline & COVsT & 156,8 & $115,8-248,4$ & 28,1 & $14,3-60,1$ & \\
\hline & MPT & 30,7 & $5,7-90,5$ & 24,9 & $7,6-37,1$ & \\
\hline 10. Edifício de escritórios & Nicotina & 0,7 & $0,4-1,7$ & ND & ND & \\
\hline$-\mathrm{RJ}$ & UV-MPI & 8,0 & $5,2-13,5$ & 4,2 & ND-7,1 & \\
\hline & Formaldeído & 40,0 & $12,2-99,7$ & 14,5 & $7,1-21,0$ & \\
\hline & Acetaldeído & 20,5 & $2,4-35,9$ & 17,0 & $8,8-27,8$ & 31 \\
\hline & COVsT & 803,2 & $304,2-1.680$ & 216,5 & $22,2-643,2$ & \\
\hline & MPT & 70,5 & $42,7-91,8$ & 81,2 & $40,0-151,0$ & \\
\hline & Benzeno & 24,9 & $11,6-34,5$ & 9,2 & $3,3-12,2$ & \\
\hline & Tolueno & 208,0 & $102,0-320,5$ & 38,4 & $8,9-60,2$ & \\
\hline & Xileno & 40,0 & $24,4-60,6$ & 14,3 & $3,7-19,9$ & \\
\hline
\end{tabular}


Tabela 2. (continuação)

\begin{tabular}{|c|c|c|c|c|c|c|}
\hline \multirow[t]{2}{*}{ Local } & \multirow[t]{2}{*}{ Poluentes } & \multicolumn{2}{|c|}{ Interno } & \multicolumn{2}{|c|}{ Externo } & \multirow[t]{2}{*}{ Referência } \\
\hline & & Média $\left(\mu \mathrm{g} / \mathrm{m}^{3}\right)$ & Faixa $\left(\mu \mathrm{g} / \mathrm{m}^{3}\right)$ & Média $\left(\mu \mathrm{g} / \mathrm{m}^{3}\right)$ & Faixa $\left(\mu \mathrm{g} / \mathrm{m}^{3}\right)$ & \\
\hline \multirow[t]{5}{*}{ 11. Escritório reformado - RJ } & Formaldeídos & 86,2 & $60,1-121,6$ & 13,9 & $0,6-18,6$ & \multirow{5}{*}{$32,33,31,34$} \\
\hline & Acetaldeídos & 25,3 & $2,35-48,5$ & 15,6 & $17,0-22,6$ & \\
\hline & COVsT & 586,1 & $300,1-1089,0$ & 134,8 & $0-215,9$ & \\
\hline & MPT & 28,7 & $42,7-91,7$ & 32,8 & $39,9-151,0$ & \\
\hline & MPI & NI & $27,4-59,5$ & NI & NI & \\
\hline \multirow{9}{*}{$\begin{array}{l}\text { 12. Escritórios, laboratório, } \\
\text { museu, escola, hospital, } \\
\text { biblioteca e residência } \\
\text { - SP }\end{array}$} & & & Máx & & Máx & \multirow{9}{*}{35} \\
\hline & Formaldeído & 29,0 & 188,0 & 9,0 & 23,0 & \\
\hline & Acetaldeído & 17,0 & 68,0 & 10,0 & 31,0 & \\
\hline & Acetona & 25,0 & 85,0 & - & 48,0 & \\
\hline & Propanal & 1,5 & 5,2 & 1,6 & 4,9 & \\
\hline & Crotonaldeído & 0,7 & 2,0 & 0,5 & 1,7 & \\
\hline & C4-isômeros & 1,4 & 3,2 & 1,5 & 4,4 & \\
\hline & Glutaraldeído & 79,0 & 121 & & & \\
\hline & Carbonílicos Totais & 48 & & 18,0 & & \\
\hline $\begin{array}{l}\text { 13. Laboratório, biblioteca, } \\
\text { hall - Araraquara, SP }\end{array}$ & Formaldeído & 54,6 & $23,0-91,8$ & & & 36 \\
\hline \multirow{4}{*}{$\begin{array}{l}\text { 14. Prédio com limpeza de } \\
\text { dutos - RJ }\end{array}$} & BTX & 33,6 & $12,4-50,2$ & 18,8 & & \multirow{4}{*}{38} \\
\hline & COVsT & 102,7 & $37,6-160,1$ & 50,8 & & \\
\hline & Formaldeído & 12,4 & $5,2-29,6$ & 3,8 & & \\
\hline & Acetaldeído & 23,3 & $12,7-33,6$ & 13,5 & & \\
\hline \multirow{4}{*}{$\begin{array}{l}\text { 15. Prédio sem limpeza de } \\
\text { dutos - RJ }\end{array}$} & BTX & 18,8 & $5,4-35,4$ & 1,3 & & \multirow{4}{*}{38} \\
\hline & COVsT & 240,8 & $105,0-633,3$ & 17,3 & & \\
\hline & Formaldeído & 22,2 & $20,6-37,5$ & 12,6 & & \\
\hline & Acetaldeído & 26,8 & $28,5-35,8$ & 3,5 & & \\
\hline \multirow{3}{*}{$\begin{array}{l}\text { 16. Prédio INCQS - } \\
\text { FIOCRUZ - RJ }\end{array}$} & COVsT & 237,1 & $82,0-620,8$ & 72,9 & & \multirow{3}{*}{39} \\
\hline & Formaldeído & 36,7 & $6,3-123,0$ & 2,9 & & \\
\hline & Acetaldeído & 40,4 & $11,9-114,8$ & 11,8 & & \\
\hline \multirow[t]{3}{*}{ 17. Creche - FIOCRUZ - RJ } & COVsT & 48,3 & $35,0-70,0$ & 50,0 & & \multirow{3}{*}{39} \\
\hline & Formaldeído & 2,2 & $0-6,8$ & 3,8 & & \\
\hline & Acetaldeído & 5,1 & $0-15,3$ & 20,0 & & \\
\hline \multirow[t]{3}{*}{ 18. Castelo - FIOCRUZ - RJ } & COVsT & 459,6 & $95,0-1.050,0$ & & & \multirow{3}{*}{39} \\
\hline & Formaldeído & 24,3 & $8,4-35,9$ & & & \\
\hline & Acetaldeído & 17,0 & $8,8-21,8$ & & & \\
\hline \multirow{2}{*}{$\begin{array}{l}\text { 19. Biomanguinhos - } \\
\text { FIOCRUZ - RJ }\end{array}$} & COVsT & & & & & \\
\hline & Formaldeído & $607,515,065$ & $0-1.150,0$ 9,4-20, & & & 39 \\
\hline 20. Biblioteca de Manguinhos & MPT & 94,7 & $35,2-150,1$ & 22,1 & $0-45,2$ & \\
\hline - FIOCRUZ - RJ & BTX & 11,7 & $5,1-21,9$ & 5,1 & $0-12,2$ & \\
\hline & COVsT & 151,6 & $60,7-267,8$ & 38,7 & $0-73,2$ & 37 \\
\hline & Formaldeído & 15,2 & $8,2-23,6$ & 4,9 & $0-13,1$ & \\
\hline & Acetaldeído & 16,9 & $5,7-31,8$ & 4,6 & $0-8,7$ & \\
\hline 21. Caixa forte de banco - RJ & COVsT & 535,6 & $321,4-1272,5$ & 207,9 & $163,2-252,7$ & \\
\hline & Formaldeído & 4,0 & $8,1-11,7$ & 6,1 & & 40,41 \\
\hline & Acetaldeído & 0,6 & $0-1,41$ & ND & & \\
\hline & MPT & 31,6 & $18,6-44,9$ & 25,2 & $17,3-33,14$ & \\
\hline 22. Gráfica - RJ & COVsT & 15192,1 & $1962,0-46154,0$ & 215,0 & $178,0-252,0$ & \\
\hline & Formaldeído & 21,3 & $5,7-37,2$ & 6,7 & $3,6-9,8$ & \\
\hline & Acetaldeído & 15,0 & $1,1-34,7$ & 7,0 & $1,3-12,8$ & \\
\hline & MPT & 32,5 & $5,3-98,0$ & 25,2 & $17,3-33,1$ & 42,43 \\
\hline & Benzeno & 2,5 & $0-13,3$ & 2,1 & $0-4,3$ & \\
\hline & Tolueno & 2921,1 & $88,5-7810,4$ & 79,3 & $66,5-92,0$ & \\
\hline & Xilenos & 18,7 & $0-325,0$ & 5,2 & $0-10,3$ & \\
\hline
\end{tabular}


Tabela 2. (continuação)

\begin{tabular}{|c|c|c|c|c|c|c|}
\hline \multirow[t]{2}{*}{ Local } & \multirow[t]{2}{*}{ Poluentes } & \multicolumn{2}{|c|}{ Interno } & \multicolumn{2}{|c|}{ Externo } & \multirow[t]{2}{*}{ Referência } \\
\hline & & Média $\left(\mu \mathrm{g} / \mathrm{m}^{3}\right)$ & Faixa $\left(\mu \mathrm{g} / \mathrm{m}^{3}\right)$ & Média $\left(\mu \mathrm{g} / \mathrm{m}^{3}\right)$ & Faixa $\left(\mu \mathrm{g} / \mathrm{m}^{3}\right)$ & \\
\hline \multirow[t]{4}{*}{ 23. Escritório - RJ } & MPT & 14,8 & $12,3-16,3$ & 15,8 & & \\
\hline & Formaldeído & 105,7 & $96,6-110,0$ & 8,8 & & \\
\hline & Acetaldeído & 0,8 & $0-1,94$ & 0 & & 51 \\
\hline & COVsT & 569,5 & $548,1-567,4$ & 216,3 & & \\
\hline \multirow[t]{6}{*}{ 24. Residências - RJ } & Benzeno & & $10,0-50,0$ & & & \\
\hline & Tolueno & & $20,0-200,0$ & & & 44,45 \\
\hline & Xileno & & $25,0-30,0$ & & & \\
\hline & COVsT & & $800-1000,0$ & & & \\
\hline & MPT & & 48,2 & & & \\
\hline & Formaldeído & & 40,0 & & & \\
\hline \multirow[t]{3}{*}{ 25. Escritórios - RJ } & Benzeno & & & $8,4 \mathrm{ppb}$ & $5,7-12,5 \mathrm{ppb}$ & 46 \\
\hline & Fumantes & $25,3 \mathrm{ppb}$ & $17,9-33,3 \mathrm{ppb}$ & & & \\
\hline & Não fumantes & $13,0 \mathrm{ppb}$ & $3,5-21,9 \mathrm{ppb}$ & & & \\
\hline \multirow[t]{2}{*}{ 26. Aeroporto - RJ } & MPT & & $16,3-283,1$ & & & \\
\hline & COVsT & & $51,0-1878,0$ & & & 47 \\
\hline $\begin{array}{l}\text { 27. Ambientes não } \\
\text { industriais - RS }\end{array}$ & Formaldeído & & $10-740 \mathrm{ppb}$ & $10 \mathrm{ppb}$ & & 48 \\
\hline $\begin{array}{l}\text { 28. Fábricas e reformadoras } \\
\text { de baterias - RJ }\end{array}$ & Chumbo & $0,302 \mathrm{mg} / \mathrm{m}^{3}$ & $\begin{array}{l}0,068 \mathrm{mg} / \mathrm{m}^{3}- \\
0,802 \mathrm{mg} / \mathrm{m}^{3}\end{array}$ & & & 49 \\
\hline
\end{tabular}

Abreviaturas: UV-PIS: partículas inaláveis em suspensão; COVsT: compostos orgânicos voláteis totais; PIS: partículas inaláveis em suspensão; MPT: material particulado total; BTX: benzeno, tolueno, xileno

Tabela 3. Limites sugeridos por órgãos nacionais e internacionais para qualidade do ar em ambientes industriais e não industriais e as concentrações médias obtidas a partir do levantamento bibliográfico da situação no Brasil

\begin{tabular}{|c|c|c|c|c|c|c|c|c|}
\hline \multirow[t]{2}{*}{ Poluente } & \multirow{2}{*}{$\begin{array}{c}\mathrm{NR}-15 \\
\left(\mathrm{mg} / \mathrm{m}^{3}\right) \\
48 \mathrm{~h} / \mathrm{sem}\end{array}$} & \multirow[t]{2}{*}{ CONAMA } & \multirow[t]{2}{*}{$\begin{array}{c}\text { Resolução } \\
176\left(\mu \mathrm{g} / \mathrm{m}^{3}\right)^{*}\end{array}$} & \multirow{2}{*}{$\begin{array}{l}\text { Aquino Neto } \\
\text { e Brickus } \\
\left(\mu \mathrm{g} / \mathrm{m}^{3}\right)^{*}\end{array}$} & \multirow[t]{2}{*}{$\begin{array}{l}\text { NIOSH } \\
\left(\mathrm{mg} / \mathrm{m}^{3}\right)\end{array}$} & \multirow[t]{2}{*}{$\begin{array}{c}\text { OSHA } \\
\left(\mathrm{mg} / \mathrm{m}^{3}\right)\end{array}$} & \multicolumn{2}{|c|}{$\begin{array}{l}\text { Faixa de concentrações } \\
\text { encontradas }\left(\mu \mathrm{g} / \mathrm{m}^{3}\right)\end{array}$} \\
\hline & & & & & & & I & $\mathbf{E}$ \\
\hline Acetaldeído & 140 & NS & NS & NS & 180 & 360 & $0,6-44,1$ & $0-33,8$ \\
\hline Benzeno & $3,19-7,97 * *$ & NS & NS & 250 & 0,325 & 3,25 & $2,5-24,9$ & $2,1-9,2$ \\
\hline COVsT & NS & NS & NS & 500 & NS & NS & $48,3-15192,1$ & $17,3-216,3$ \\
\hline Formaldeído & 2,3 & NS & NS & 100 & 0,02 & 0,93 & $2,2-105,7$ & $2,9-14,5$ \\
\hline MPT & NS & 80 & 80 & 80 & NS & NS & $14,8-94,7$ & $15,8-81,2$ \\
\hline Nicotina & NS & NS & NS & NS & 3,37 & 3,37 & $0,3-1,7$ & $0-0,06$ \\
\hline Tolueno & 290 & NS & NS & 250 & 375 & 766 & $208,0-2921,4$ & $37,6-79,3$ \\
\hline Xilenos & 340 & NS & NS & 250 & 435 & 435 & $18,7-496,3$ & $5,2-14,3$ \\
\hline
\end{tabular}

* ambientes internos; ** casos especiais; NS: não sugerido

jam coerentes, são utilizados apenas como recomendação, assim como os padrões internacionais.

De acordo com a Tabela 3, observa-se que em muitos casos, principalmente para COVsT, foram ultrapassados os limites sugeridos nacionais $\left(500 \mu \mathrm{g} / \mathrm{m}^{3}\right)^{7}$ e internacionais $\left(300 \mu \mathrm{g} / \mathrm{m}^{3}\right)^{29}$. Em alguns casos também formaldeído, MPT, tolueno e xilenos ultrapassaram os limites recomendados.

\section{CONCLUSÕES}

Com base nos dados expostos pode-se observar que são poucos os estudos no Brasil referentes à QAI. Os dados são preocupantes, por isso, deve ser feito um esforço no sentido de aumentar o número de pesquisas nessa área para todo o Brasil. Os dados então obtidos poderiam ser utilizados para determinar limites compatíveis com nosso clima, economia e cultura.

\section{AGRADECIMENTOS}

Ao CNPq, à CAPES (PIBIC) e à FUJB pelo suporte financeiro.

\section{REFERÊNCIAS}

1. WHO (World Health Organization); Report WHO Meeting, Nordlingen, 8-11 junho, 1982.

2. Brickus, L. S. R.; Aquino Neto, F. R.; Quim. Nova 1999, 22, 65.

3. Jones, A. P.; Atmos. Environ. 1999, 33, 4535.

4. Gioda, A.; Aquino Neto, F. R.; Cadernos Saúde Pública/FIOCRUZ, no prelo. 
5. Ministério da Saúde, Portaria 3523 de 28 de agosto de 1998.

6. ANVISA, Agência Nacional de Vigilância Sanitária, Resolução 176 de 24 de outubro de 2000.

7. Aquino Neto, F. R.; Brickus, L. S.R.; Rev. Brasindoor 1999, 3, 4.

8. Lei $n^{\circ} 6514,22$ de dezembro de 1977, Segurança e Medicina do Trabalho, Ministério do Trabalho, 26ª ed., Editora Atlas: São Paulo, 1994.

9. CONAMA, Conselho Nacional do Meio Ambiente, Resolução n ${ }^{\circ} 03$ de 28/ 06/1990.

10. NIOSH, (National Institute of Occupational and Safety Health); NIOSH pocket guide to chemical hazards, Washington DC, USA, US Gov Printing Office, 1994

11. USEPA (U.S. Environmental Protection Agency); Technical assistance document for sampling and analysis of toxic organic compounds in ambient air. [EPA-60018-90-005] Washington D. C. EPA, method TO-11, 1990.

12. OSHA; Occupational exposure to hazardous chemicals in laboratories, Laboratory Standard 29 CRF 1910.1450, 1990.

13. WHO (World Health Organization); The right to health indoor air, Report WHO Meeting, Bilthoven, Netherlands, 15-17 may, 2000.

14. Grosjean, D.; Miguel, A. H.; Tavares, T. M.; Atmos. Environ. 1990, 24B, 101.

15. Pomaleski, D. R. M.; Miguel A. H.; Resumos da $14^{a}$ Reunião Anual da Sociedade Brasileira de Química, Caxambú, Brasil, 1991.

16. Vasconcellos, P. C.; Miguel, A. H.; Resumos da $16^{a}$ Reunião Anual da Sociedade Brasileira de Química, Caxambú, Brasil, 1993.

17. Miguel, A. H.; Allen, A. G.; Vasconcellos, P. C.; Aquino Neto, F. R.; Cardoso, J. N.; Proc. Indoor Air 1993, 3, 229.

18. Miguel, A. H.; Aquino Neto, F. R.; Cardozo, J. N.; Vasconcellos, P. C.; Pereira, A. S.; Marquez, K. S. G.; Environ. Sci. Technol. 1995, 29, 338.

19. Miguel, A. H.; Pedreira Filho, W. R.; Allen, A. G.; Indoor Built Environ. $\mathbf{1 9 9 5}, 4,355$.

20. Allen, A. G.; Miguel, A. H.; Atmos. Environ. 1995, 29, 3519.

21. Santos, C. Y. M.; Aquino Neto, F. R.; Cardoso, J. N.; Indoor Built Environ. 1997, 6, 168

22. Silva, M. A.; Brickus, L. S. R.; Aquino Neto, F. R.; Moreira, J. C.; Resumos da $21^{a}$ Reunião Anual da Sociedade Brasileira de Química, Poços de Caldas, Brasil, 1998.

23. Ugucione, C.; Cardoso, A. A.; Gomes Neto, J. A.; Resumos da $24^{a}$ Reunião Anual da Sociedade Brasileira de Química, Poços de Caldas, Brasil, 2001.

24. Oliveira, C. J. F.; Cardoso, J. N.; Aquino Neto, F. R.; Resumos do VII Encontro Regional de Química/ Sociedade Brasileira de Química, Rio de Janeiro, Brasil, 1998.

25. Oliveira, C. J. F.; Cardoso, J. N.; Aquino Neto, F. R.; Resumos da $21^{a}$ Reunião Anual da Sociedade Brasileira de Química, Poços de Caldas, Brasil, 1998.

26. Leite, F.; Brickus, L. S. R.; Costa, M. F. B.; Moreira, J. C.; Aquino Neto, F. R.; International Conference On Environment and Occupational Cancer in Developing Countries, Rio de Janeiro, Brasil, 1998.

27. Brickus, L. S. R.; Moreira, J. C.; Resumos da $21^{a}$ Reunião Anual da Sociedade Brasileira de Química, Poços de Caldas, Brasil, 1998.
28. Brickus, L. S. R.; de Oliveira, C. J. F.; Cardoso, J. N.; Aquino Neto, F. R.; Resumos da 21 Reunião Anual da Sociedade Brasileira de Química, Poços de Caldas, Brasil, 1998.

29. Molhave L.; Clausen G.; The $7^{\text {th }}$ International Conference On Indoor Air Quality and Climate, 1996.

30. Brickus, L. S. R.; Aquino Neto, F. R.; Cardoso, J. N.; Resumos da $22^{a}$ Reunião Anual da Sociedade Brasileira de Química, Poços de Caldas, Brasil, 1999.

31. Brickus, L. S. R.; Cardoso, J. N.; Aquino Neto, F. R.; Environ. Sci. Technol. 1998, 32, 3485 .

32. Brickus, L. D.; Cardoso, J. N.; Aquino Neto, F. R.; Proc. Healthy Buildings 1997.

33. Brickus, L. S. R.; Aquino Neto, F. R.; Cardoso, J. N.; Resumos do VII Encontro Regional de Química/ Sociedade Brasileira de Química, Rio de Janeiro, Brasil, 1998

34. Aquino Neto, F. R.; Cardoso, J. N.; Brickus, L. S. R.; Proc. Indoor Air, Edinburgh, Scotland, 1999.

35. Pires, M.; Montero, L.; Carvalho, L. R. F.; Quim. Nova 1999, 22, 487.

36. Pereira, E. A.; Cardoso, A. A.; Dasgupta, P. K.; Quim. Nova 2001, 24, 443.

37. Brickus, L. S. R.; Aquino Neto, F. R.; Moreira, J. C.; Siqueira, L. F. G.; The $8^{\text {th }}$ International Conference on Indoor Air Quality and Climate, Scotland, 1999.

38. Brickus, L. S. R.; Costa, M. F. B.; Tome, P. A.; Machado, J. H.; Moreira, J. C.; Resumos do $1^{\circ}$ Seminário Saúde Ambiente no Processo de Desenvolvimento, Rio de Janeiro, Brasil, 1998.

39. Brickus, L. S. R.; Neves, F. L.; Moreira, J. C.; Resumos da $1^{a}$ Bienal de Pesquisa da FIOCRUZ, Rio de Janeiro, Brasil, 1998.

40. Carneiro, F. P.; Aquino Neto, F. R.; Rev. Brasindoor 2001, V, 18.

41. Carneiro, F. P.; Tese de Mestrado, Universidade Federal do Rio de Janeiro, Brasil, 2001.

42. Gioda, A.; Aquino Neto, F. R.; Rev. Brasindoor 2001, V, 20.

43. Gioda, A.; Aquino Neto, F. R.; Indoor Built Environ. 2002, 11, 302.

44. Almeida, S. M.; Corrêa, M. A. P.; Brickus, L. S. R.; Aquino Neto, F. R.; Moreira, J. C.; Resumos do $10^{\circ}$ Encontro Nacional de Química Analítica, Santa Maria, Brasil, 1999.

45. Almeida, S. M.; Neves, F. L.; Brickus, L. S. R.; Aquino Neto, F. R.; Moreira, J. C.; Rev. Brasindoor 2001, V, 24

46. Costa, M. F. B.; Moreira, J. C.; Rev. Brasindoor 2001, V, 21.

47. Silveira, M. G.; Brickus, L. S. R.; Fernando, S. G. L.; Moreira, J. C.; Rev. Brasindoor 2001, $V, 26$.

48. Bortolli, A. A.; Pires, M.; Rev. Brasindoor 2001, V, 31.

49. Mattos, R. C. O. C.; Silva, C. R. S.; Araújo, U. C.; Moreira, M. F. R.; Gomes, R. A. A.; Santos, L. C. S.; Quitério, S. L.; Caldeira, C.; Nogueira, S. M.; Moreira, J. C.; Rev. Brasindoor 2001, V, 33.

50. Parreira, F. V.; Carvalho, C. R.; Cardeal, Z. L.; Rev. Brasindoor 2001, V, 28.

51. Santos, C. Y. M.; Carneiro, F. P.; Gioda, A.; Aquino Neto, F. R.; Laudo Técnico nº 151/2001, LADETEC/IQ/UFRJ, 2001. 